\title{
Inhibitory Effect of Ubiquinone on Biosynthesis of Aldosterone in Rat Adrenal in vitro
}

\author{
AKIRA KUMAGAI, KAZUHIKo NISHINO, \\ TAKEKAZU KIMM, ATSUSHI SHIMOMURA, \\ MASAHISA NANAHOSHI AND YUICHI YAMAMURA
}

The 3rd Department of Internal Medicine

Medical School of Osaka University, Osaka

\begin{abstract}
Synopsis
The effect of ubiquinone $\left(\mathrm{Co}_{7}\right)$ was investigated in vitro on the biosynthesis of aldosterone from progesterone- $-4-{ }^{14} \mathrm{C}$ and corticosterone- $1,2-{ }^{3} \mathrm{H}$ using quartered rat adrenals. When progesterone was incubated with the adrenal tissue, the following metabolites could be obtained: 11-desoxycorticosterone, $11 \beta$-hydroxyprogesterone, corticosterone, 18-hydroxycorticosterone and aldoster one. Ubiquinone inhibited the production of 18-hydroxycorticosterone and aldosterone with the resultant accumulation of corticosterone. This inhibitory effect of ubiquinone on the formation of aldosterone was more clearly observed when corticosterone was used as the substrate.

These results suggest that ubiquinone inhibits the activity of 18-hydroxylase in the adrenal, resulting in the reduction of aldosterone biosynthesis.
\end{abstract}

Increased evidence of the importance of aldosterone in the pathogenesis of various diseases has made it attractive to look for its inhibitors. Two kinds of inhibitors have been reported: 1) the inhibition of aldosterone effect on sodium resorption by distal tubule of kindney and 2) the inhibition of biosynthesis or secretion of aldosterone by adrenal cortex. Spirolactones (Kagawa et al., 1957; Liddle, 1957) and progesterone (Landau et al., 1955; 1958) belong to these compounds of the first category. They do not inhibit the secretion of aldosterone or other adrenal corticoids (Coppage et al., 1960). On the contrary, a compensatory increase in aldosterone secretion rate has been reported following administration of spirolactones (Singer, 1959).

Most of the inhibitors of the second category, paticularly pyridine derivatives, (Raman et al., 1966) cannot be used for aldosterone suppression in humans because of toxicity or

Received for publication April 8, 1970. poor specificity. Only heparin and some related polysulfated polysaccharides show great promise of clinical use (Beiglbock et al., 1952; Schlatmann et al., 1960; Glaz et al., 1964; Sharma et al., 1967).

In search of the glomerrlotropic factor (GTF) in the pineal gland, Farrell et al. (Farrell, 1960) found that certain extracts of pineal tissue contained an aldosterone inhibitor in lipid fractions which suppressed the activity of GTF for the secretion of aldosterone. Recently (1965), Fabre et al., succeeded in purifying and identifying the inhibitor as ubiquinone. We had a case of Conn's syndrome (Kumagai, 1967) in which hypokalemia and other accompanying symptoms were suprisingly improved by the treatment with ubiquinone.

In the present study, the effect of ubiquinone on the conversion of progesterone and corticosterone to 18-hydroxycorticosterone and aldosterone has been investigated in the rat adrenal. 


\section{Materials and Methods}

Progesterone-4- ${ }^{14} \mathrm{C}$ (sp. act. $21.7 \mathrm{mc} / \mathrm{m}$ mole) and corticosterone-1, $2-{ }^{3} \mathrm{H}$ (sp. act. $0.46 \mathrm{mc} / \mathrm{m}$ mole) were obtained from Daiichi Kagaku Company and Tracer Laboratory respectively. Ubiquinone $\left(\mathrm{Co} \mathrm{Q}_{7}\right)$ was kindly supplied by Takeda Pharmaceutical Company. Authentic 18-hydroxycorticosterone was synthesized by Dr. W. Nagata of Shionogi Pharmaceutical Co. NADPH was purchased from Sigma Chemical Company.

Female Sprague-Dawley rats weighing 120 to $150 \mathrm{~g}$ were used for the source of adrenal tissue. Immediately after removal, the adrenals were placed on ice and carefully cut into quarters with a thin razor blade. About $200 \mathrm{mg}$ of these fresh quartered adrenals were added to each of the incubation flasks. Besides the adrenal tissue, each incubation flask contained $1 \mu \mathrm{c}$ of progesterone- $4-{ }^{14} \mathrm{C}$ for corticosterone- $1,2-{ }^{3} \mathrm{H}$ as the substrate, $1 \mu M 0.1 \mathrm{~m} l \mathrm{NADPH}$ as a hydrogen donor, 1.0 or $0.1 \mathrm{mg}$ of $\mathrm{Co}_{7}$ or blank solvent, and KrebsRinger bicarbonate buffer containing $200 \mathrm{mg} \%$ of glucose to a total volume of $5 \mathrm{ml}$.

Incubations were done in air at $38^{\circ} \mathrm{C}$ for $3 \mathrm{hr}$ in a Warburg metabolic shaker. After incubation $100 \mu \mathrm{g}$ of nonlabeled steroid substrate (progesterone or corticosterone) was added and the incubated tissue with the incubation medium was homogenized in a PotterElvehjem glass homogenizer. The steroids were extracted from the homogenate with three- $20 \mathrm{~m} l$ portions of chloroform. The pooled extract was washed with $0.1 \mathrm{~N} \mathrm{NaOH}, 0.1 \mathrm{~N} \mathrm{NaHCO}_{3}$ and water, and was evaporated to dryness in a rotary evaporator. Partial purification of the steroids was carried out by running through Florisil column following Eik-Nes's method (Eik-Nes et al., 1953). The steroids were eluted in the fraction of $25 \%$ methanol in chloroform.

The eluate was rechromatographed on a Amberlite IRC-50 column $\left(1 \times 67 \mathrm{~cm}, 39^{\circ} \mathrm{C}\right)$ with solvent of methanol : ethanol : water $(3: 9: 9)$ according to Seki's method (Seki and Matsumoto., 1967). Forty drops (approximately $l \mathrm{~m} l$ ) were collected in a tube with a fraction collector of drop count type. Progesterone, 11-desoxycorticosterone (DOC), 11-hydroxyprogesterone, corticosterone + aldosterone, and 18hydroxycorticosterone were satisfactorily separated from each other. The radioactivity of the steroid fractions was counted with Packard TriCarb liquid scintillation counter. As corticosterone and aldosterone were eluted into the same fractions by the column, the separation was carried out by paper chromatography of Mattox system (Mattox and Lewbart, 1959). Radioactivity of ${ }^{14} \mathrm{C}$-steroids was measured directly on the paper by actigraph, while that of ${ }^{3} \mathrm{H}$ steroids was determined by Packard Tri-Carb liquid scintillation counter after elution with ethanol. All samples were run in duplicate and the data was presented as the mean of the duplicate.

\section{Results}

When progesterone-4- ${ }^{14} \mathrm{C}$ was incubated with the adrenal tissue, the following metabolites could be detected by the column chromatography: 18-hydroxycorticosterone, corticosterone + aldosterone, 11-hydroxyprogesterone, DOC and progesterone. The formation of the last three steroids were very little affected by the addition of $1.0 \mathrm{mg}$ of ubiquinone in vitro. On the other hand, the peak of 18-hydroxycorticosterone was greatly reduced by ubiquinone, while that of corticosterone + aldosterone were increased by ubiquinone (Fig. 1). Paper chromatography of
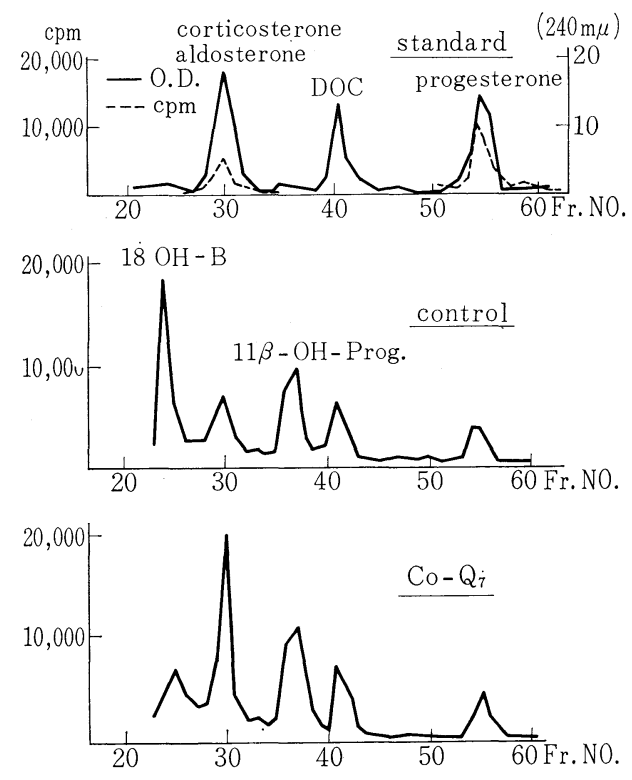

Fig. 1. Elution pattern of column chromatography. Typical examples of the column chromatography of some authorized materials of steroid are shown on top. The other curves are isotopic patterns of metabolites from progesterone- $4-{ }^{14} \mathrm{C}$ after incubation of rat adrenal slices without $\operatorname{Co} Q_{7}$ (Control) and with $\mathrm{Co} \mathrm{Q}_{7}$. Incubation were done under the condition described in Materials and Methods. 
the fraction of corticosterone + aldosterone provided an evidence of the fact that this increase was attributable only to corticosterone and the production of 18-hydroxycorticosterone and aldosterone was clearly reduced by ubiquinone (Fig. 2, 3).

When corticosterone- $1,2-{ }^{3} \mathrm{H}$ was used as the substrate, only two peaks of steroids, 18hydroxycorticosterone and corticosterone + aldosterone, were detected by the column chromatography. The peak of 18-hydroxycorticosterone was decreased by 0.1 and 1.0 $\mathrm{mg}$ of ubiquinone and the effect seemed to be dose-dependent (Fig. 4). The second peak was unaffected by ubiquinone, but paper chromatography of the fraction showed that the production of aldosterone and 18-hydroxycorticosterone was decreased by $1.0 \mathrm{mg}$ of ubiquinone (Fig. 5, 6).

If the ratios of the formed metabolites to the added substrate after the incubation are calculated, the effect and site of action of ubiquinone can be more clearly shown. The ratio of corticosterone to progesterone was increased by 4 times by ubiquinone, while the ratio of 18-hydroxycorticosterone + aldosterone to progesterone was decreased to less than half of that of the control (Table 1). Both ratios of 18-hydroxycorticosterone and of aldosterone to corticosterone were decreased by the addition of ubquinone (Table 2). Therefore, it is reasonably concluded that

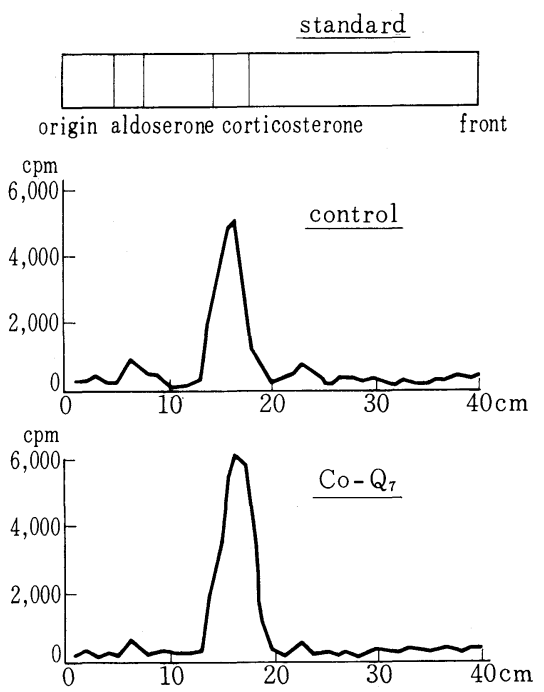

Fig. 2. Elution pattern of paperchromatography. Typical examples of the paperchromatography of authorized materials of aldosterone and corticosterone are shown on top. The other curves are isotopic patterns of paperchromatography to divide aldosterone and corticosterone of Fig. 1.

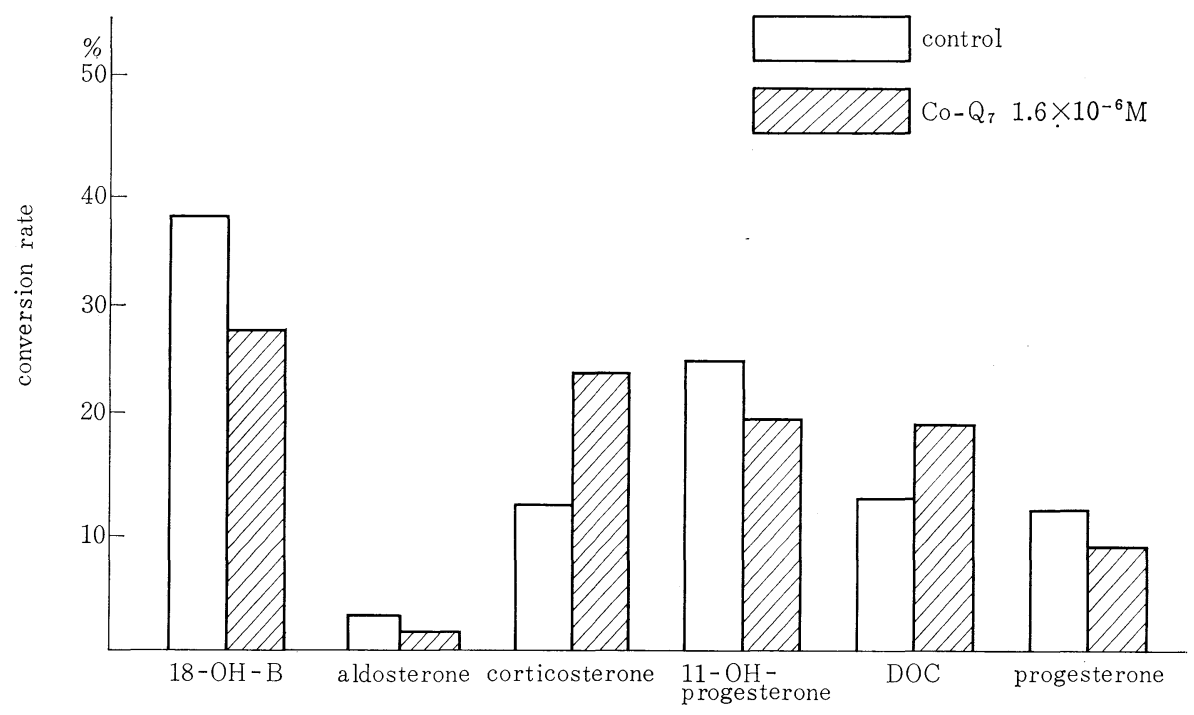

Fig. 3. Effects of $\mathrm{Co}_{7}$ on the conversion rate of progesterone-4- ${ }^{14} \mathrm{C}$ into several steroids. 


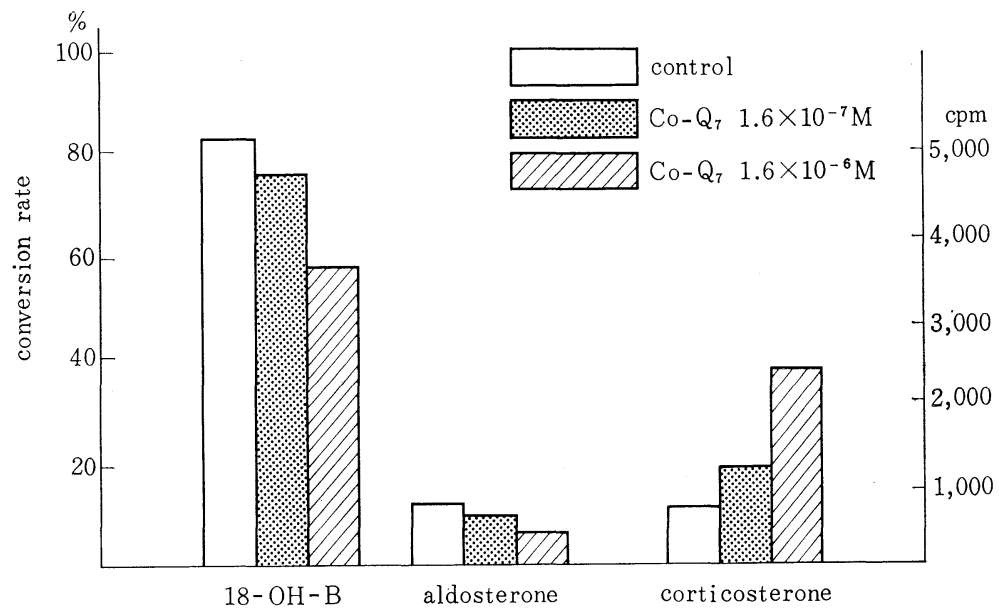

Fig. 4. Effects of $\mathrm{Co} \mathrm{Q}_{7}$ on the conversion rate of corticosterone-1,2- ${ }^{3} \mathrm{H}$ into 18-hydroxycorticosterone and aldosterone.
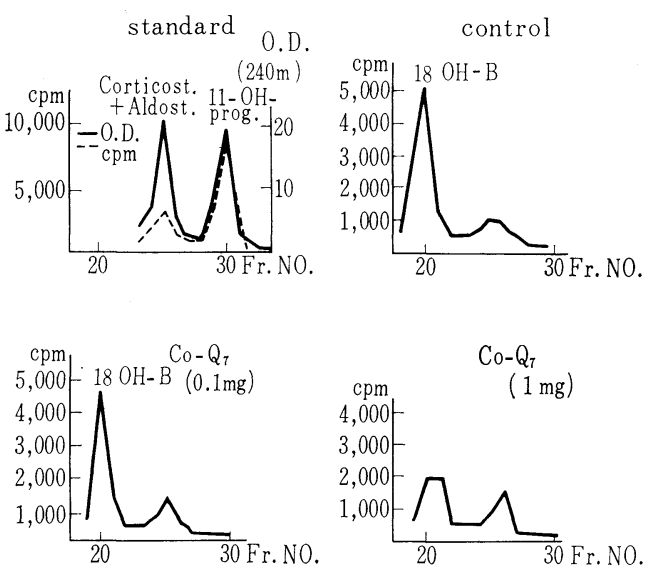

Fig. 5. Elution pattern of column chromatography. Typical examples of the column chromatography of corticosterone, aldosterone and 11-OHprogesterone are shown (upper, left). The other curves are isotopic patterns of metabolites from corticosterone-1,2- ${ }^{3} \mathrm{H}$ after incubation of rat adrenal slices without $\mathrm{Co}_{7} \mathrm{Q}_{7}$ (Control) and with $\mathrm{Co}_{7} 0.1 \mathrm{mg}$ and $1.0 \mathrm{mg}$ respectively. Incubation were under the condition described in Materials and Methods.


Fig. 6. Elution pattern of paper chromatography. Typical examples of the paperchromatography of authorized materials of aldosterone and corticosterone are shown on top. The other curves are isotopic patterns of paperchromatography to divide aldosterone and corticosterone of Fig. 3 . 
Table 1. Ratio of ${ }^{14} \mathrm{C}$-steroid Metabolites over ${ }^{14} \mathrm{C}$-Progesterone after incubation

\begin{tabular}{|c|c|c|}
\hline${ }^{14} \mathrm{C}$-steroid & Additive & Ratio \\
\hline Corticost./ & None & 0.9 \\
\hline Progest. & Co Q & 3.7 \\
\hline 18-OH Corticost.+Aldost./ & None & 3.4 \\
\hline Progest. & Co $\mathrm{Q}$ & 1.5 \\
\hline
\end{tabular}

Ratios were calculated from the radioactive counts of the steroids of Fig. 3.

Table 2. Ratio of ${ }^{3} \mathrm{H}$-steroid Metabolites over ${ }^{3} \mathrm{H}-$ corticosterone after incubation. Ratios were calculated from the radioactive counts of the steroids of Fig. 4

\begin{tabular}{cll}
\hline${ }^{3} \mathrm{H}$-steroid & Additive & Ratio \\
\hline $18-\mathrm{OH}$ corticost./ & None & 7.33 \\
Corticost. & Co Q & 3.95 \\
\hline Aldost./ & None & 0.67 \\
Corticost. & Co Q & 0.23 \\
\hline
\end{tabular}

ubiquinone exerts its inhibitory effect on the metabolic step immediately after corticosterone, leading to an accumulation of corticosterone and a reduction of its metabolites.

\section{Discussion}

In 1960, Farrell (Farrell, 1960) reported that certain extracts of pineal tissue administered intravenously inhibited steroidgenesis in the dog. Five years later, the same author and his coworkers, demonstrated (Fabre et al., 1965) that ubiquinone, a constituent of the lipid fraction of beef pineal gland, was mainly responsible for this inhibition. Clinical trial of ubiquinone has been given in our hands for the control of hyperaldosteronism (Kumagai, 1967). In a case of Conn's syndrome the symptoms such as hypokalemia, headache, weakness, etc. were surprisingly improved by treatment with ubiquinone $100 \mathrm{mg} /$ day for a week. Many cases of heart failure responded fairly well to the administration of ubiquinone (Yamamura et al., 1967). Encouraged by these clinical observations as well as Farrell's reports, we have undertaken the present ex- periment to see the effect of ubiquinone on the in vitro biosynthesis of aldosterone by the rat adrenal tissue.

It is generally accepted that 18-hydroxycorticosterone is the main intermediate on the biosynthetic pathway between corticosterone and aldosterone though the details are still remained unknown. NADPH is required as an indispensable cofactor this metabolic process as in other steps of steroid hydroxylation. A recent report of Greengard et al (Greengard et al., 1967) indicated that cytochrome $\mathrm{P}-450$, a component of mixed function oxidase system requiring NADPH, is involved in the biosynthesis of aldosterone from corticosterone by adrenal mitochondria. As ubiquinone is found in adrenal mitochondria, it is quite concevable that it can act as a hydrogen trap for NADPH to inhibit the adrenal systems by which aldosterone is produced. However, if this is true, every step of steroid hydroxylation should be equally inhibited. Since the inhibition by ubiquinone is rather specific for 18hydroxylation and NADPH is added exogenously to the incubation system in the present experiment, this possibility for the mechanism of inhibition can be excluded. Other factors such as calcium (Buddecke and Dryzenick, 1962) or renin-angiotension system (Schlatmann et al., 1964; Sealey et al., 1967) which were proposed for the action of heparin, can also be excluded for the mechanism of ubiquinone in the present in vitro experiment. Thus, at present, the direct inhibition of 18hydroxylase in the adrenal cell would be the most probable mechanism of inhibitory action of ubiquinone.

The clinical application of ubiquinone is very interesting because of its potential use to combat hyperaldosteronism of various causes. Spirolactones are now the first choice in the treatment of secondary hyperaldosteronism. The diuretic effect of spirolactones is sometimes quite dramatic for edema of secondary hyperaldosteronism. But, as they have no direct effect on the secretion or production of aldosterone by the adrenal cortex, the effect is 
only transitory in most cases of primary aldosteronism. In this regard, ubiquinone looks very promising because of its low toxicity and high specificity for the inhibition of aldosterone production.

\section{Acknowledgement}

The authors wish to thank Drs. T. Seki and K. Matsumoto of Osaka University for their excellent technical assistance and criticism on chromatographic analyses of steroids. Thanks are also due to Dr. W. Nagata of Shionogi Pharmaceutical Company for the synthesis of 18-hydroxycorticosterone, and to Takeda Pharmaceutical Company for the gift of ubiquinone.

\section{References}

Beiglbock, W., K. Sivkel and E. Clotten (1952). Muench. Med. Wichschr. 94, 410. Buddecke, E. and R. Dryzenick (1962), Z. Physiol. Chem. 327, 49.

Coppage, W. S., Jr. and G. W. Liddle (1960). Ann. N. Y. Acad. Sci. 88, 815.

Eik-Nes, K., D. H. Nelson and L. T. Samuels (1953). J. Clin. Endocrinol. 13, 1280.

Farrell, G. L., (1960). Circulation 21. 1009.

Fabre, L. F., R. C. Banks, W. M. McIsaac and G. Farrell (1965). Am. J. Physiol. 208, 1275.

Glaz, E. and K. Sugar, (1964). Endocrinology 74, 159.
Greengard, P., S. Psychoyos and H. H. Tallan (1967). Arch. Biochem. 121, 298.

Kagawa, C. M., J. A. Cella, and C. G. Van Arman (1957). Science 126, 1015.

Kumagai, A., (1967). Proc. 17th General Assembly of Japan. Med. Congress 1, 521.

Liddle, G. W., (1957). Science 126, 1016.

Landau, R. L., D. M. Bergnestal, K. Lugibihl and M. E. Kasht (1955). J. Clin. Endocrinol. 15, 1194 .

Landau, R. L. and K. Lugibihl (1958). Ibid. 18, 1237.

Mattox, V. R. and M. L. Lewbart (1959). Ibid. 19, 1151.

Raman, P. S., D. C. Sharma and R. I. Dorfman (1966). Biochem. 5, 1795.

Singer, B., (1959). Endocrinolgy 65, 512.

Schlatmann, J. R., A. F. M., A. P. Jansen, H. Prenen and C. L. H. Majoor (1960). Lancet 278, 317.

Sharma, D. C., C. A. Nerenberg and R. I. Dorfman (1967). Biochem. 6, 3472.

Seki, T. and K. Matsumoto (1967). J. Chromatogr. 27, 423.

Schlatmann, J. R., A. F. M., A. P Jansen, H. Prenen, J. K. van der Korse and C. L. H. Majoor (1964). J. Clin. Endocrinol. 24, 35. Sealey, J. E., J. N. Gerten, J. G. G. Ledingham and J. H. Laragh (1967). Ibid. 27, 699.

Yamamura, Y., T. Ishiyama, Y. Morita and T. Yamagami (1967). Clinic all-Round 17, 1564. 